\begin{tabular}{|c|c|c|}
\cline { 2 - 3 } & REVISTA SABERES APUDEP & Volumen 3 Número 2 \\
\hline
\end{tabular}

\title{
CONSERVACIÓN DE TORTUGAS LORA (Lepidochelys olivacea), EN PLAYA MATA OSCURA, VERAGUAS, PACÍFICO DE PANAMÁ
}

\author{
CONSERVATION OF TURTLES LORA (Lepidochelys olivacea), IN PLAYA MATA \\ OSCURA, VERAGUAS, PACIFIC OF PANAMA
}

\author{
Rodríguez, Belsy \\ Universidad de Panamá Centro Regional Universitario de Colón. Biología Ambiental \\ belsyr98@gmail.com \\ Mónica Contreras \\ Universidad de Panamá Centro Regional Universitario de Colón. Zoología de \\ Vertebrados \\ monicanuzhat@gmail.com \\ https://orcid.org/0000-0003-0972-6951
}

\section{RESUMEN}

Lepidochelys olivacea (Eschscholtz, 1829) es una tortuga marina con un ciclo de vida en el cual las hembras nidifican en playas de arena, desde las cuales los neonatos emergen e ingresan al ambiente marino para continuar su desarrollo. En Panamá, algunas organizaciones sin fines de lucro promueven estrategias para conservar estos chelonios a la luz de las normativas vigentes. Este articulo documenta la actividad reproductiva de tortugas loras en Playa Mata Oscura, Veraguas, Pacifico de Panamá acompañada de actividades de conservación, entre los meses de julio a diciembre de 2018. Se reporta anidada solitaria, con avistaron diecisiete nidos que fueron relocalizados al vivero de la Fundación Agua y Tierra. La media para el tamaño de la nidada fue de 61.05 huevos. Con un éxito de eclosión de nidos relocalizados de $73.69 \%$; estos en su totalidad lograron remerger y fueron liberados en buenas condiciones físicas.

Palabras Clave: Lepidochelys olivacea, Actividad reproductiva, Playa Mata Oscura, Panamá. 


\begin{tabular}{|c|c|c|}
\cline { 2 - 3 } & REVISTA SABERES APUDEP & Volumen 3 Número 2 \\
\hline
\end{tabular}

\begin{abstract}
Lepidochelys olivacea (Eschscholtz, 1829) is a sea turtle with a life cycle in which females' nest on sandy beaches, from which hatchlings emerge and enter the marine environment to continue their development. In Panama, some nonprofit organizations promote strategies to conserve these chelonios in light of current regulations. This article documents the reproductive activity of parrot turtles in Playa Mata Oscura, Veraguas, Pacific of Panama accompanied by conservation activities, between the months of July to December 2018. It is reported nesting solitary, with seventeen nests reported that were relocated to the nursery of the Fundación Agua y Tierra. The mean for clutch size was 61.05 eggs. With a hatching success of relocated nests of $73.69 \%$; These in their entirety managed to remerge and were released in good physical condition.
\end{abstract}

KEYWORDS: Lepidochelys olivacea, Reproductive activity, Playa Mata Oscura, Panama.

\title{
INTRODUCCIÓN
}

Lepidochelys olivacea (Eschscholtz, 1829) es una de las 5 especies de tortugas marinas reportadas para Panamá. El comportamiento altamente gregario de estas tortugas, las ubica en un estado vulnerable (UICN), dada la extracción indiscriminada de sus huevos en las playas (Ministerio de Ambiente Panamá, 2017) y el alto número de muertes accidentales por los barcos pesqueros (Orrego, 2005)

Las hembras adultas pueden realizar anidaciones solitarias o en arribadas, anidación masiva o sincronizada de cientos o miles de tortugas durante varios días consecutivos, (Viejobueno Muñoz y Randall Arauz, 2014). Sin embargo, Poco se ha registrado sobre la anidación de esta especie en la costa del Pacífico de Panamá, por lo que es fundamental priorizar esfuerzos para definir playas de mayor importancia y establecer protección de las mismas (Ley 8 de 2008). 


\begin{tabular}{|c|c|c|}
\cline { 2 - 3 } & REVISTA SABERES APUDEP & Volumen 3 Número 2 \\
\hline
\end{tabular}

Los movimientos migratorios de las tortugas lora están menos estudiados que en otras tortugas marinas, pero se sabe que involucra aguas costeras de más de 80 países (Chary Tala, 2016), entre estos: México, Nicaragua, Costa Rica y Panamá (VásquezBultrón, 2012).

En Panamá, algunas fundaciones sin fines de lucro, promueven estrategias para conservar especies de tortugas marinas a la luz de las normativas vigentes, como es el caso de la Fundación Agua y Tierra (FUNDAT), que desde el año 2011, promueve el Programa de Conservación de Tortugas Marinas en la Península de Azuero; incorporando el voluntariado y un programa de marcaje de tortugas.

En la actualidad el proyecto ha iniciado un proceso de certificación de turismo sostenible comunitario con la Autoridad de Turismo de Panamá (ATP), y mantiene firme sus objetivos de protección y conservación.

Las investigaciones científicas que se desarrollan al margen de actividades de conservación de tortugas marinas por esta fundación deben ser documentadas, por lo que en este articulo publicaremos los resultados de un estudio sobre la actividad reproductiva de tortugas lora, también conocida como tortuga golfina, temporada 2018. empleando como indicadores los descriptores cuantitativos de la fase final del período de incubación debido a que se considera uno de etapas de mayor vulnerabilidad (Boulon, 2000).

\section{MATERIALES Y MÉTODO}

Para estudiar la fase de incubación de L. olivacea se implementó un monitoreo intensivo durante toda la temporada de anidación mediante el cual se tomaron los datos desde el momento de la oviposición hasta el análisis del nido luego de la eclosión. 


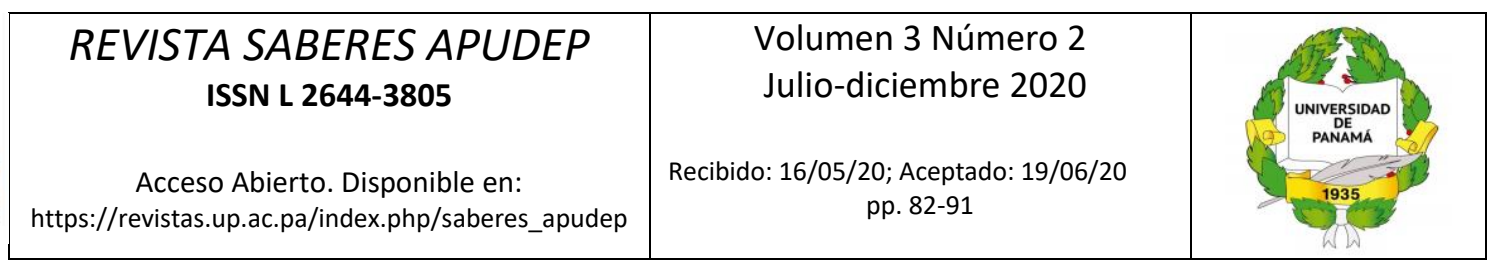

\section{ÁREA DE ESTUDIO.}

Este estudio se realizó entre los meses de julio a diciembre , temporada de anidación de tortuga lora, de 2018 en Playa Mata Oscura, ubicada en el Corregimiento de Quebro, Distrito de Mariato, Provincia de Veraguas, Pacífico Panameño, localizado entre 7.46456 de Latitud Norte ( $7^{\circ} 27^{\prime} 52$ ') y -80.93189 de Longitud Oeste (W $\left.80^{\circ} 55^{\prime} 54^{\prime \prime}\right)$; Zona Sur de Veraguas, declarada por la Autoridad de los Recursos Acuáticos de Panamá (ARAP), en el 2008, como Zona Especial de Manejo Marino Costera (ZEMMC). Con temperatura que oscila entre $\quad 25^{\circ} \mathrm{C}$ y 34 en el día y $27^{\circ} \mathrm{C}$ y $25^{\circ} \mathrm{C}$ noche. Con lluvias frecuentes entre mayo y diciembre y una humedad de 92 $\%$. En esta playa se han registrado arribadas de cuatro especies de tortugas marinas: Tortugas Lora (L. olivacea), Tortugas Verde (C. mydas), Tortuga Carey (E. imbricata), Tortuga Canal (D. coriacea) (Rodríguez, 2018). (Figura1).
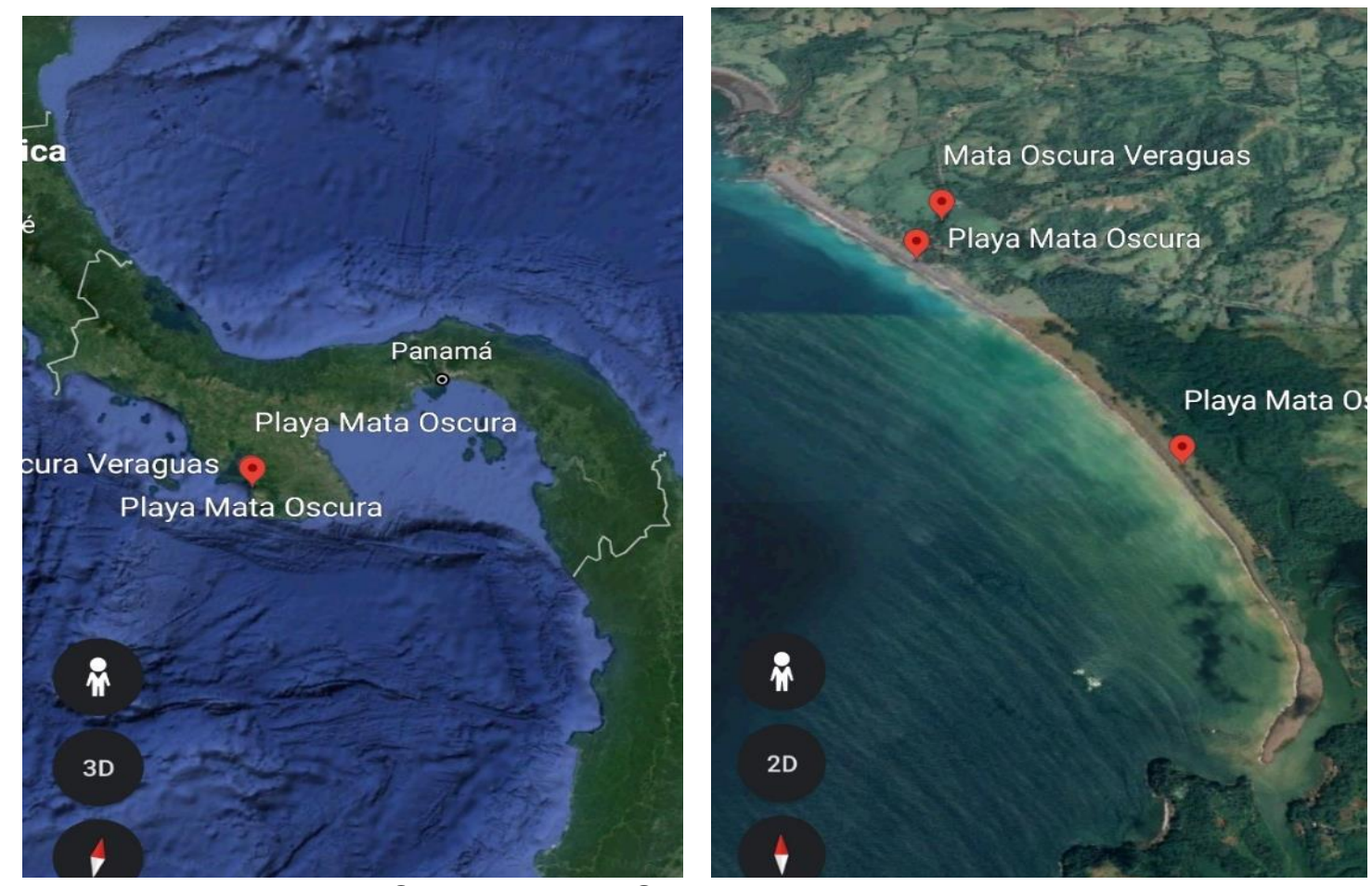

Figura 1 Playa Mata Oscura, Zona Sur de Veraguas. Vista satelital. Fuente Google Earth (2019). 
REVISTA SABERES APUDEP

ISSN L 2644-3805

Acceso Abierto. Disponible en:

https://revistas.up.ac.pa/index.php/saberes_apudep
Volumen 3 Número 2

Julio-diciembre 2020

Recibido: 16/05/20; Aceptado: 19/06/20 pp. 82-91

\section{TÉCNICAS PARA LA OBSERVACIÓN Y REGISTRO DE NIDOS DE L. olivacea}

Patrullaje: Para registrar los sitios de anidamiento, se recorrían diariamente $3 \mathrm{~km}$ de playa, con una duración de dos horas. Al encontrar rastro de recorrido de la tortuga, se localiza el nido, se marca con barillas.
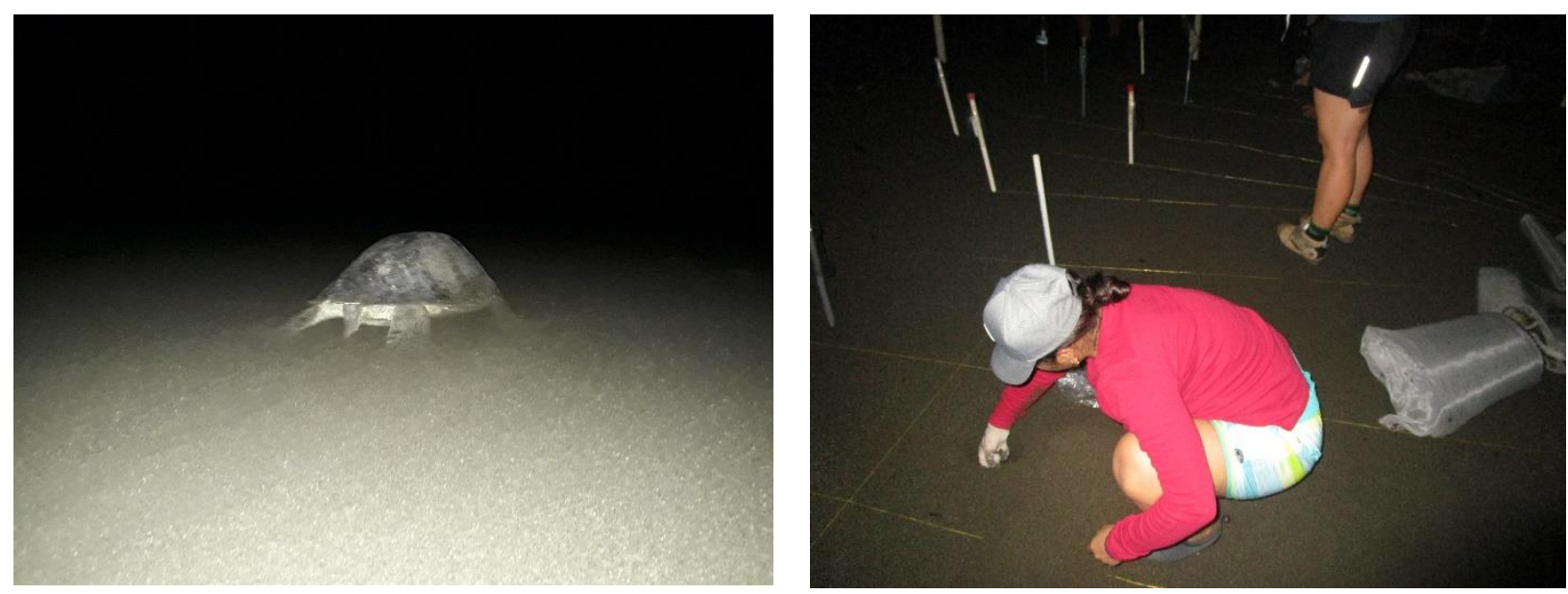

Figura 2. Localización de nidos L. olivacea. Fotografía B. Rodríguez, 2018.

Actividad Reproductiva: los indicadores fueron $\mathrm{N}^{\circ}$ de huevos por nidada (Rees, Saad y Jony, 2005), éxito de eclosión y éxito de emergencia. El éxito de la incubación, se determinó a través de: éxito de eclosión (porcentaje de crías que eclosionan o rompen la cascara respecto al número total de huevos depositados) y emergencia (número de crías vivas y muertas que eclosionaron y alcanzaron la superficie de la playa (Miller, 2000)

Éxito de eclosión $=$ Huevos eclosionados $X 100$ Nidada

Éxito de emergencia $=$ Huevos eclosionados $X 100$ \# Crías vivas al mar 


\begin{tabular}{|c|c|c|}
\cline { 2 - 3 } & REVISTA SABERES APUDEP & Volumen 3 Número 2 \\
\hline
\end{tabular}

\section{RESULTADOS Y DISCUSION}

Se identificaron diecisiete nidos de tortugas lora (Lepidochelys olivacea), para la temporada 2018. Siendo el mes de diciembre el mes de mayor actividad reproductiva con un total de 10 eventos de anidación. El tamaño promedio de la nidada fue de 61 . 05 huevos, con una variación desde un número de 25 hasta un máximo de 109 (tabla 1). Estos resultados son comparables con los de Viejobueno y Arauz, quienes en 16 años de monitoreo en una Playa localizado en sur Pacifico de Costa Rica, reportan nidada promedio anual de 96.7 huevos.

Tabla 1 Tamaño de Nidada Lepidochelys olivacea

\begin{tabular}{|l|l|l|l|l|l|}
\hline \multirow{2}{*}{ Temporada } & \multicolumn{5}{|l|}{ Actividad reproductiva } \\
\cline { 2 - 6 } & $N^{\circ} \mathrm{N}$ & TH & TN & TMin. & TMax. \\
\hline 2018 & 17 & 1038 & 61.05 & 25 & 109 \\
\hline
\end{tabular}

No N Nidos avistados TH Total de huevos TN Tamaño Nidada TMin. Tamaño Mínimo TMax. Tamaño Máximo

El éxito de eclosión de tortugas lora en el vivero fue de $73.69 \%$; El mayor éxito se alcanzó en el mes de diciembre 85\% (332 huevos eclosionados/389); mientras que en el mes de octubre se registró el menor éxito de (80 huevos eclosionados). En todos los casos se alcanzó el $100 \%$ de emergencia y las crías fueron liberadas en buenas condiciones físicas (Figura 3). 


\begin{tabular}{|c|c|c|}
\cline { 2 - 3 } & REVISTA SABERES APUDEP & Volumen 3 Número 2 \\
\hline
\end{tabular}

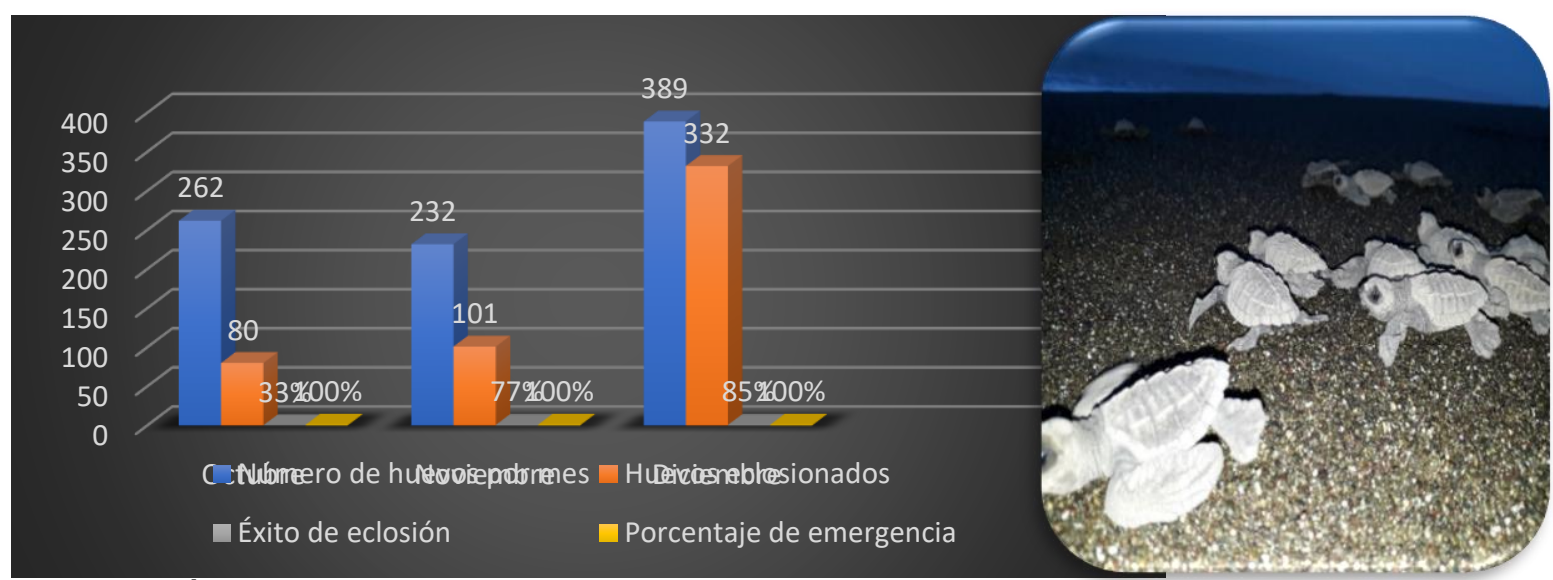

Figura 3. Éxito de eclosión L. olivacea. Vivero Fundación Agua y Tierra FUNDAT

El éxito de eclosión de tortuga lora en el vivero reportado en este estudio, coinciden con los reportados por Malmierca (2018), 72,94\% y los reportados por Viejobueno Muñoz y Arauz, 2014, quienes reportan 76.54\% de huevos que llegan a eclosionar. Las nidadas de tortugas marinas típicamente tienen un alto éxito de eclosión (80\% o más), aunque factores externos como la depredación, cambios ambientales o infecciones microbianas influyen sobre este parámetro (Miller, 1997; Julia et al., 2006).

Durante los meses octubre y noviembre de ejecución del proyecto, el vivero de tortuga lora fue afectado por dos oleajes que removieron la posición de los nidos, acompañado de depredación por Canis lupus familiaris, por lo que se reforzaron las medidas de seguridad del vivero, dado que la actividad de anidación de la tortuga lora ocurre en una forma descrita como anidación solitaria.

Existe controversia acerca de la utilidad del traslado de nidos y la eliminación de criaderos de protección in situ, porque una de las causas que puede ocasionar un menor éxito de eclosión es el manejo de los nidos (Ehrenfeld, 1995; Pritchard, 1995, citado por Sandoval, 2008). Ya que, según Enciso (1991), citado por Sandoval, 2017, el movimiento de los huevos es una de las causas de la inhibición en el desarrollo embrionario y su mortalidad. 


\begin{tabular}{|c|c|c|}
\cline { 2 - 3 } & REVISTA SABERES APUDEP & Volumen 3 Número 2 \\
\hline \\
\hline
\end{tabular}

La principal técnica de manejo usada en Playa Mata Oscura por la Fundación Agua y Tierra para la conservación de tortugas marinas (Figura 4), ha sido la operación de viveros. Los cuales, a su vez, se traducen en instrumentos de educación ambiental, permitiendo a los miembros de la comunidad involucrase en los esfuerzos de conservación, con la participación de estudiantes voluntarios con estudios universitarios, se hace el vínculo universidad comunidad. Estas actividades pueden asegurar, además, un estilo de vida en las comunidades con entradas económicas, disminuyendo la presión antrópica y de otros depredadores de extracción de huevos de tortugas marinas.
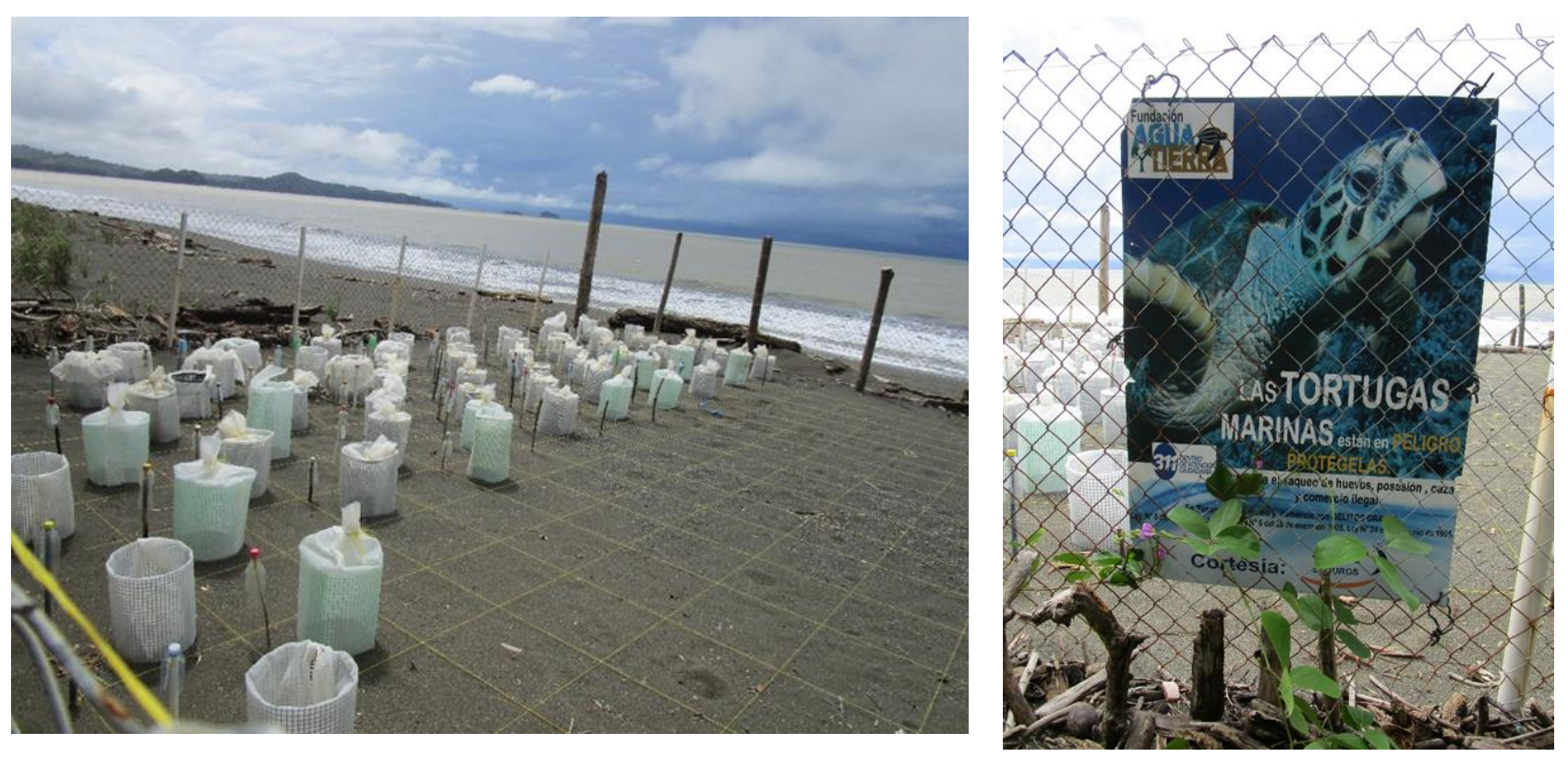

Figura 4. Viivero de tortugas marinas de Fundación Agua y Tierra." Las Tortugas Marinas están en peligro, protégelas. Fotografía por B. Rodríguez, 2018. 


\begin{tabular}{|c|c|c|}
\cline { 2 - 3 } & REVISTA SABERES APUDEP & Volumen 3 Número 2 \\
\hline
\end{tabular}

La actividad de cosecha de huevos de tortugas para la venta está prohibida en todo el territorio nacional de Panamá, a excepción de Isla cañas, donde la colecta de huevos para subsistencia está permitida, Resolución "CIT-COP6-2013-R1 sobre excepciones bajo el artículo IV ( 3 A y B) para la cosecha de subsistencia de huevos de Lepidochelys olivacea en Panamá (Ministerio de Ambiente, 2017).

\section{CONCLUSIÓN}

Los descriptores de la fase final de la incubación reportados en este estudio constituyen indicadores para evaluar el éxito reproductivo de las tortugas lora en Playa Mata oscura; lo que puede ser considerado como una estrategia para evaluar la efectividad de la estrategia de conservación utilizada en tortugas lora por la Fundación Agua y Tierra, Panamá.

\section{AGRADECIMIENTO}

Se le agradece al cuerpo de voluntarios y al director de la Fundación Agua y Tierra, Jacinto Rodríguez, por todo su apoyo y trabajo.

\section{REFERENCIAS BIBLIOGRÁFICAS}

Boulon, R.H. (2000): Reducción de las Amenazas a los Huevos y las Crías. Protección in situ. En: Research and management techniques for the conservation of sea turtles (K.L. Eckert, K.A. Bjorndal, F.A. Abreu-Grobois y M. Donnelly, eds.), IUCN/SSC Marine turtles specialist group publication No. 4, $235 \mathrm{pp}$.

Chary tala, G. (2016). Ficha de Antecedentes de la especie Lepidochelys olivacea. Ministerio de Medio Ambiente RCE.

Engelbrecht, L. (2015). Tortugas: fundamentales para el Ecosistema Marino. Tortugas Marinas.

Julia Azanza R., Ruisanchez Carrasco, Y., Ibarra, M., Ruiz Urquiola, R., César Y. Castellanos I, Ríos Tamayo, D y Colectivo de estudiantes de la Facultad de Biología 2. (2006). Indicadores del Éxito Reproductivo de la Tortuga Verde (Chelonia mydas) en Tres Playas de La Península de Guanahacabibes, Pinar Del Río, Cuba. Rev. Invest. Mar. 27(1):69-78 


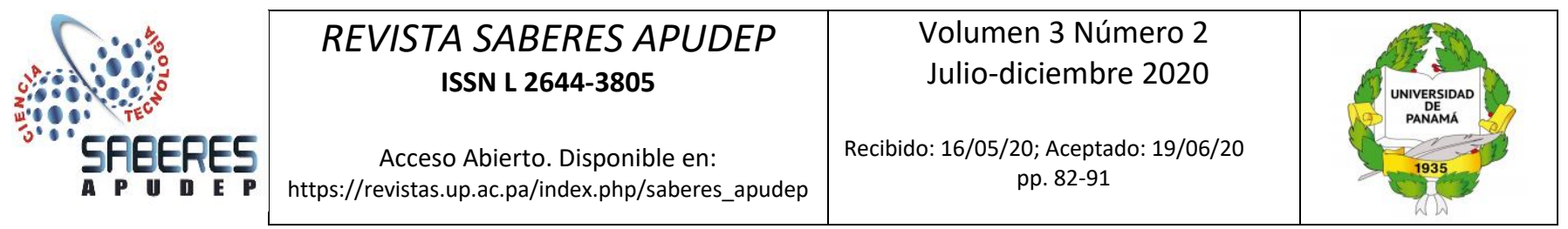

Ministerio de Ambiente Panamá (2017). Diagnóstico de la situación de tortugas marinas en Panamá y Plan de Acción 2017-20121.

http://marviva.net/sites/default/files/documentos/diagnostico situacion tortugas marinas pa nama y pan conservacion 17-04.pdf

Miller, J. D. (2000). Determinación del Tamaño de la Nidada y el Éxito de Eclosión. En Manual de técnicas de investigación y manejo para la conservación de las tortugas marinas, UICN/CSE Grupo de especialistas en tortugas marinas. p.143.

Miller, J.D. (1997): Reproduction in sea turtles. In: The Biology of Sea Turtles (P.L. Lutz y J.A. Musick, eds). CRC, Boca Raton, pp: 51-83.

Orego, C., 2005. Causas antrópicas y naturales en la mortalidad de las tortugas baula (Dermochelys coriacea), lora (Lepidochelys olivacea) y verde (Chelonia mydas agassizi), en la costa Pacífica de Costa Rica. Tesis Ms. Universidad Nacional. Heredia, Costa Rica. $29 \mathrm{pp}$.

Pita Fernández S, Pértega Díaz S. Asociación de variables cualitativas: Test X2. Cad Aten Primaria 2004; 11: 236-239.

Pritchard, P. (1969) Studies of the systematics and reproductive cycles of the Genus Lepidochelys. Ph.D. dissertation, University of Florida, FL.

Rees, A., Saad, A., \& Jony, M. (2005). Clutch size and hatching success of green turtle nests in Syria during 2004. In A. Demetropoulos \& O. Turkozan (Eds.), Proceedings of the Second Mediterranean Conference on Marine Turtles (pp. 158-161). Kemer: Turkey.

Rodríguez, Jacinto 2018. Entrevista personal sobre avistamiento de Tortugas Lora (Lepidochelys olivacea), Tortugas Verde (Chelonia mydas), Tortuga Carey (Eretmochelys imbricata), Tortuga Canal (Dermochelys coriacea) en playa Mata Oscura.

Sandoval Espinoza, S. (2008). Pronóstico de la temperatura de los nidos de tortuga golfina (Lepidochelys olivacea) en función de la temperatura ambiente, la profundidad y el calor metabólico. Instituto Politécnico Nacional, La paz, B.C.S, México.

Sandoval Ramírez, J. L. (2017.) Influencia de factores ambientales sobre el éxito de incubación de la tortuga golfina (Lepidochelys olivacea) en condiciones de vivero en el estado de Guerrero, México. (Tesis). Centro de Investigación Científica y de Educación Superior de Ensenada, Baja California. México.

UICN. Lista Roja de Especies Amenazadas. https://www.iucnredlist.org/

Viejobueno Muñoz S y Arauz, R. (2014). Conservación y actividad reproductiva de tortuga (Lepidochelys olivacea) en la Playa de Anidación Solitaria Punta Barco, pacífico Sur de Costa Rica. Recomendaciones de Manejo de 16 años de monitoreo. Revista Biológica Tropical. 63, 383-394 